\title{
Patient specific dosimetric calculations obtained by planar images and Monte Carlo simulation in ${ }^{111}$ In octreotide therapy
}

\author{
Argyrou M, Andreou M, Lagopati N, Baka I, Vamvakas I and Lyra M* \\ Radiation Physics Unit, A’ Radiology Department, Kapodistrian University of Athens, Vas. Sophias Ave, Athens, Greece
}

\begin{abstract}
The hedge of a declared accuracy in dosimetric calculations in modern nuclear medicine clinical routine is very important and absolutely obligated. Patient specific dosimetry calculations help the physician to optimize the planning of the treatment, avoid side effects to healthy tissue and assign administered dose to treatment results, so the optimization of this method is very desirable. In this study, scintigraphic data from 22 patients, diagnosed for neuroendocrine tumors in liver, received therapeutic dose of ${ }^{111} \mathrm{In}$ octreotide, were used in order to estimate the absorbed doses from radiopharmaceutical volume distributions and absorbed doses in the lesions and in critical organs, employing the MIRD schema, as well as the Monte Carlo simulation, for a comparison of the individualized palliative treatment absorbed doses with the one obtained by mathematical code pathway.
\end{abstract}

\section{Introduction}

In recent years, a number of new developments in targeted therapies using radiolabeled compounds have emerged. Use of molecular imaging to design personalized therapy gives a great challenge to medical physicists to be involved in research of molecular targeted radionuclide therapies and especially in the dosimetric part of them.

The idea of a radionuclide used in therapy is based on the desire to link a radionuclide which has a high linear energy transfer associated with its decay products such as Auger electrons, $\beta$-particles or $\alpha$-particles to a biologically active molecule that can be directed to a tumour site. $\beta$-emitting radionuclides, rich in neutrons, have been produced in reactors, though few of them are best produced via charged particle reactions. In order for these radionuclides to be effective as anticancer agents, they must either could be located at or near the cancer cells (high LET particles) or near the DNA in the nucleus (Auger emitters).

${ }^{111} \mathrm{In}$ is produced in cyclotron by ${ }^{112} \mathrm{Cd}$ collision with protons of energy $2.8 \mathrm{MeV}$ according to the nuclear reaction ${ }^{112} \mathrm{Cd}(\mathrm{p}, 2 \mathrm{n}){ }^{111} \mathrm{In}$. The radioactive ${ }^{111} \mathrm{In}$ decays to ${ }^{112} \mathrm{Cd}$ with physical half-life time of 2.83 days. Purity of the final product of ${ }^{111} \mathrm{In}$ is affected by the undesired isotopes ${ }^{110 \mathrm{~m}} \mathrm{In},{ }^{110} \mathrm{In}$ and ${ }^{114 \mathrm{~m}}$ In that are not possible to spare from ${ }^{111} \mathrm{In}$ due to the similar chemical characteristics of these isotopes. However, the isotopes ${ }^{110 \mathrm{~m}} \mathrm{In}$ and ${ }^{110} \mathrm{In}$, do not affect dosimetry of radioisotopes labeled with ${ }^{111}$ In, because these undesired isotopes have minor presence and short half-life time ( $4.9 \mathrm{~h}$ and $1.1 \mathrm{~h}$, respectively). On the contrary, ${ }^{114 \mathrm{~m}} \mathrm{In}$ that is produced from ${ }^{114} \mathrm{Cd}$ according to a $(\mathrm{p}, \mathrm{n})$ nuclear reaction, has 49.51 days half-life time and decays with internal transition (96.9\%) and electron capture (3.2\%) with emission of photons at 192, 558 and 725 $\mathrm{keV} .{ }^{114 \mathrm{~m}} \mathrm{In}$ affects dosimetry due to its long half-life time.

Human - peptide receptor scintigraphy started with the in vivo demonstration of somatostatin receptor-positive tumours in patients using a radioiodinated somatostatin analogue. Somatostatin is an endogenous neuropeptide that acts as a regulator of growth hormone secretion [1] and it is present in many neurons and endocrine cells, mainly in the brain and in the gastrointestinal tract, having an inhibitory effect on growth hormone secretion.

Starting in the 1990s, attempts at treatment with radiolabeled somatostatin analogues were undertaken in patients with inoperable and/or metastasized neuroendocrine tumours. Improvements in the peptides used, with higher receptor affinity, in the radionuclides that were applied, with $\beta$ and Auger e- emissions, together with precautions to limit the radiation dose to the kidneys and the bone marrow, led to better results with a virtually negligible percentage of serious adverse events.

Because the radioiodinated somatostatin analogue that was first used for imaging [ $\left.{ }^{123} \mathrm{I}, \mathrm{Tyr}^{3}\right]$ octreotide, had several drawbacks, soon a chelated and ${ }^{111}$ In-labeled somatostatin analogue, [ $\left.{ }^{111} \mathrm{In}-\mathrm{DTPA}\right]$ octreotide, was developed and is commercially available. ${ }^{111} \mathrm{In}$ DTPA-d-Phe ${ }^{1}$ - octreotide (also known as ${ }^{111}$ In - pentetreotide or ${ }^{111} \mathrm{In}$-octreotide) is a peptide composed of eight amino acids and is an analogue of the active part of the peptide hormone somatostatin [2]. ${ }^{111}$ In - pentetreotide specifically binds to receptors for tissues where the cell surfaces contain these receptors in higher than physiological density [3], as a consequence of disease. Thus, there is an over expression of the somatostatin receptors at the surface of the nerve endocrine tumour cancer cells. ${ }^{111} \mathrm{In}$, with a half-life of $67.92 \mathrm{~h}$, it disintegrates by Auger and internal conversion electrons and emits maximum gamma photons of 0.171 and $0.246 \mathrm{MeV}$.

*Correspondence to: Lyra M, Radiation Physics Unit, A' Radiology Department, Kapodistrian University of Athens, Vas. Sophias Ave, Athens, Greece, E-mail: mlyra@med.uoa.gr

Received: October 10, 2018; Accepted: October 19, 2018; Published: October 22,2018 
It is one of the radioisotopes of choice for liver lesions radionuclide therapy. By its gamma emissions, the scintigraphic follow-up imaging is possible and offers valuable information of the radionuclide distribution.

Firstly, it has been indicated for the use in diagnosis of somatostatin receptor-bearing tumors, such as pancreatic (GEP) tumors, small cell lung cancer and breast cancer, by aiding in their localization. Tumors that bear neither somatostatin receptors nor sufficient receptors density are not visualized [4]. The clinical impact is that in vivo receptor scintigraphy uses radiolabeled peptides for the localization of tumours and their metastases, but also peptide receptor radiotherapy of tumours has been emerged as a serious treatment option.

In palliative treatment use, the radiopharmaceutical entrance into the tumour cell and its destructive effect to DNA by emission of Auger and internal conversion electrons are exploited. Patient specific dosimetry calculations help the physician to optimize the planning of the treatment, avoid side effects to healthy tissue and assign administered dose to treatment results. The aim of this study was to estimate the absorbed dose in tumor and healthy tissues using anterior and posterior planar scintigrams [5-10], of patients, diagnosed for neuroendocrine tumours in liver, received therapeutic dose of ${ }^{111} \mathrm{In}$ octreotide, via intrahepatic catheterization. Individualized data calculations for each patient for an internal dosimetry in nuclear medicine unfortunately raise the disadvantage of attenuation and scatter corrections lack and organ overlay.

A comparison of the individualized palliative treatment absorbed doses, by planar images data with Monte Carlo N-Particle Code (MCNPX) absorbed doses calculations was adopted in order to indicate step by step the procedures that are valuable in the improvement of in vivo measurements. MIRD schema was employed to estimate the individualized absorbed doses for each patient, from radiopharmaceutical volume distributions and absorbed doses in the lesion area as well as in critical organs, concerning kidneys, spleen, liver and pancreas. In parallel, Monte Carlo simulation, based on a random number system was undergone, in order to verge on the real experiment, meaning the radiopharmaceutical infusion in the areas, previously mentioned.

\section{Method}

In this study, 22 patients, with histologically confirmed neuroendocrine tumors located in liver and normal kidney function were infused therapeutic dose of ${ }^{111}$ In octreotide, with mean activity of $4500 \mathrm{MBq}$, via intrahepatic catheterization, which is well established technique in our Institution in hepatocellular carcinoma and neuroendocrine tumors treatments

The infusion of ${ }^{111} \mathrm{In}$ - pentetreotide (Octreoscan, Mallinckrodt, Petten, The Netherlands) was performed directly to the patient's liver through a port, attached to the hepatic artery. This is proved to be the best method to give better uptake of the radiopharmaceutical by the tumor. The hepatic artery port makes the therapy more comfortable for the patient as in this way the hepatic artery angiography catheterization, at each therapeutic session, is avoided. In order to protect their kidneys, they previously received the appropriate amino acids.

\section{Scintigraphic calculations of absorbed doses}

Planar scintigraphy was performed after radiopharmaceutical infusion, through hepatic arterial port, 24 and at $48 \mathrm{~h}$ post infusion, for the patients with liver lesions. Anterior and posterior planar images were obtained using an APEX SPX4 El Scint gamma camera.
For the needs of methodology of this work, Gamma - camera was calibrated in order to estimate source organ activity considering count rate, patient's body diameter and source organ size. The patients were imaged by a medium energy all-purpose collimator, $20 \%$ energy window centered at a $247-\mathrm{keV}^{111}$ In photo peak and 1 minute time to acquire each and every image.

A set of measurements were carried out in order to convert the count rate that was measured from planar images to activity at patient organs. A cubic tank of volume equal to $[0.1 \times 0.3 \times 0.3] \mathrm{m}^{3}$ was filled with water and was placed on the examination table and simulated patient's body [11-13]. Ten milliliters vials were placed at the centre of the cubic phantom consecutively, filled with ${ }^{111}$ In radionuclide.

Planar scintigrams of each vial were obtained with ${ }^{111}$ In activity varying from 200 to $2000 \mathrm{MBq}[13,14]$.

A linear fit function was applied to correlate the measured count rate with source organ activity.

\section{$\mathrm{R}=0,19 \mathrm{~A}+0,41$}

where R: count rate (in kilocounts $\cdot \mathrm{s}^{-1}$ ) and $\mathbf{A}$ : activity (in $\mathrm{MBq}$ ).

Radiation emitted from organ source is absorbed by patient's body. It is necessary to correct measured count rate with the transmission factor. Transmission factor T can be calculated if we know patient's body diameter, using the function:

$$
\mathrm{T}=\mathrm{e}^{-\mu \mathrm{L}}
$$

Where,

L: patient's body diameter (anterior-posterior diameter) and

$\boldsymbol{\mu}$ : linear absorbent coefficient for water. study.

The value for $\mathbf{L}$ can be obtained by patient's computed tomography

Also, radiation is absorbed by the source organ. A correction factor $\mathbf{f}$ is applied due to this phenomenon. Factor $\mathbf{f}$ can be calculated from the function:

$$
\mathbf{f}=[(\mu \mathrm{d} / 2) / \sinh (\mu \mathrm{d} / 2)]
$$

Where, $\mathbf{d}$ : anterior-posterior size of the source target (also obtained by the CT processing) [15].

Considering the factors $\mathrm{T}, \mathrm{f}$ and gamma - camera sensitivity E, measured count rate was converted to activity $\mathbf{A}$ according to the function:

$$
A=\left(R_{\text {ant }} R_{\text {post }} / T\right)^{1 / 2}(\mathbf{f} / \mathbf{E})
$$

Where

$\mathrm{R}_{\text {ant }}$ : the measured count rate from anterior image and

$\mathrm{R}_{\text {pos }}$ : the measured count rate from the posterior image [16].

The count rates were measured with manually drawn regions of interest for liver, spleen, kidneys and tumor as shown in Figure 1. Background count rate was measured close to regions of interest and a simple background subtraction method was performed. Activity was calculated from the planar anterior and posterior images $4 \mathrm{hrs}$ after radiopharmaceutical infusion and at 24 and 48 hours later. Activity was calculated for the tumor, liver, kidneys and spleen. Curves of activity as a function of time were drawn for each source organ. The area between each curve and time axis is the cumulative activity [17]. 


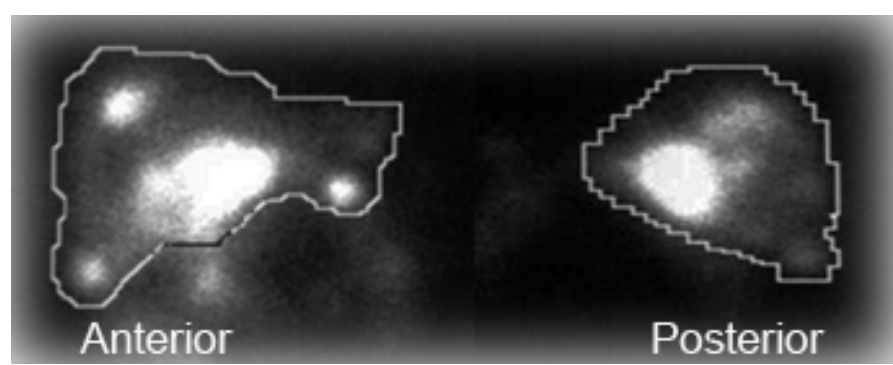

Figure 1. Planar images of liver with ROIs drawn for the activity calculation

Cumulative activity $\boldsymbol{A}$ is measured in MBq.hr and was calculated for each source organ. The residence time $t$ was calculated for each source organ using the function:

$$
\mathbf{t}=A_{c} / A_{0}
$$

Where, $\boldsymbol{A}_{\boldsymbol{0}}$ is the total infused activity

This method is appropriate for patient specific dosimetric calculations as residence times are calculated from patient's scintigraphic image.

The MIRD schema, proposed by the Medical Internal Radiation Dose (MIRD) Committee, is widely accepted for absorbed dose calculations in the scale of human organs (i.e. greater than a centimeter) $[9,10,18]$. The MIRD schema attempts to calculate the mean absorbed dose, assuming an average tissue deposition of energy and a uniform distribution of the radiopharmaceutical. The dose is calculated for the target region $(T)$, by summing up the contribution of each source region (S) to the target and the contribution of the target region itself. Any region containing activity greater than the average concentration of activity in the total body is accepted as source region. It is assumed that non - penetrating radiation (beta particles, Auger electrons, internal conversion electrons and photons below $13 \mathrm{keV}$ ) is absorbed only if it is emitted within the target region [19]. On the other hand, penetrating radiation emitted by all source regions, including radiation emitted by the target region itself, contributes to the absorbed dose to the target region. The mean absorbed dose can be roughly estimated, due to major limitations in absorbed dose calculations; these come from the inherent difficulty in measuring radioactivity inside the body, as well as from the use of standard generalized biokinetic models, that may deviate considerably from the suitable for certain patient's size and physiology.

In order to calculate the absorbed dose to the target organ, it is necessary to calculate the cumulative source activity $\boldsymbol{A}_{\boldsymbol{c}}$ in the source organ, which is the sum of all the radioactive decays over the time interval of interest, using the following equation:

$$
A_{c}=A_{0} \cdot t
$$

Where $\mathbf{A}_{0}$ : administered activity and $\mathbf{t}$ : residence time.

\section{Monte Carlo simulation}

Monte Carlo (MC) simulation gives us the opportunity to receive fast and accurate patient-specific dosimetric calculations.

For the MC simulation, two patients, one male and one female of similar weights $(70 \mathrm{~kg})$, diagnosed for neuroendocrine tumors located in liver, were considered to be as representative cases, to be used in the simulation process.

The Monte Carlo geometries, used in Monte Carlo simulation input files, were prepared in order to approach the patient's anatomy. In
Figure 2, the input file for the MCNPX code (Monte Carlo N - Particle Extended Code) is presented.

For the preparation of the input file, the tumor was considered to be a sphere of $2-3 \mathrm{~cm}$ radius, internally of a spherical form simulating the liver. The spherical cells inside the tumor were defined from 0 to $1 \mathrm{~cm}$ distance from the centre of the point radioactive source, every $0.2 \mathrm{~cm}$, from 1 to $4 \mathrm{~cm}$, every $0.5 \mathrm{~cm}$ and from 4 to $10 \mathrm{~cm}$ every $1 \mathrm{~cm}$. The radioactive source was considered as a point source, (as mentioned with the SDEF command), and it identifies with the center of the tumor (POS=0 0 0). One million events were counted.

Afterwards, using the previously measured, Regions of Interest, we tried to compare, from a geometrical point of view, the results obtained by Monte Carlo simulation and those from MIRD schema. Hence a comparison for individualized palliative treatment absorbed doses, by planar images data and Monte Carlo simulation, for the case of liver lesions is presented.

Actually, we manually selected the volumes around the tumor, in order to estimate the count rates in the areas, choosing the same depths $(0-10 \mathrm{~cm})$, as we did, employing the MCNPX code, so as to achieve an objective comparison between the results.

\section{Result}

The MIRD schema was used in order to calculate patient absorbed dose. The calculation is fast; dose estimation is accurate and patient specific, as residence times are calculated separately for each patient from his own planar images. A Microsoft Excel calculation sheet has been developed so that count rate was converted to activity in a simple way. Measured count rate, patient's body diameter, administered activity and source organ size is entered to the program and cumulative activity is automatically calculated. Cumulative activity curves were then drawn for the tumor, kidneys, spleen, liver and pancreas for each patient and residence times were then calculated for each organ.

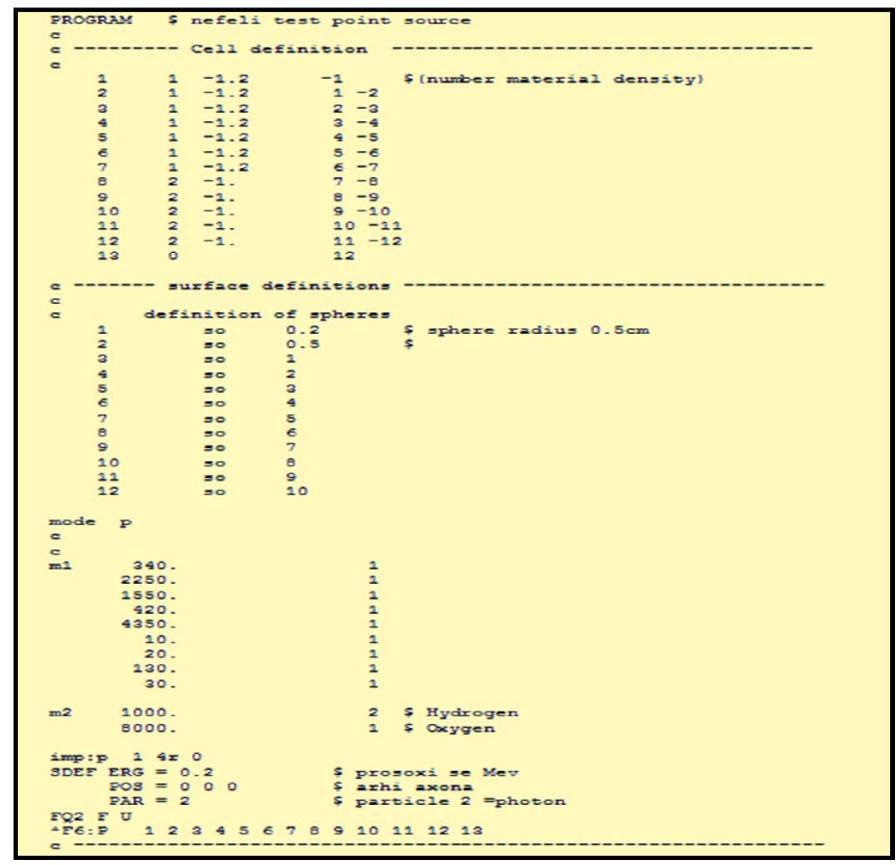

Figure 2. The MCNP-X input file for the estimation of absorbed doses in the case of tumor lesions located in the liver 
Indicatively for some of our patients, activity curves were drawn for each organ as shown in Figures 3a-3e. Patient 3 appears to have the total activity accumulated to kidneys and not to the tumor probably because the amount of somatostatins is insufficient.

Taking into account the residence time and following the MIRD schema the tumor mean absorbed dose was found ranged from 2.5 to $18.4 \mathrm{mGy} / \mathrm{MBq}$, depending on the lesion size and is presented in Figure 4. A deviation of about $7 \%$ in comparison with Monte Carlo results (Figure 5) was found, where a linearity of the absorption of radiation was observed, with a maximum distribution of the dose at the center of the tumor and a gradual decrease runaway of it.

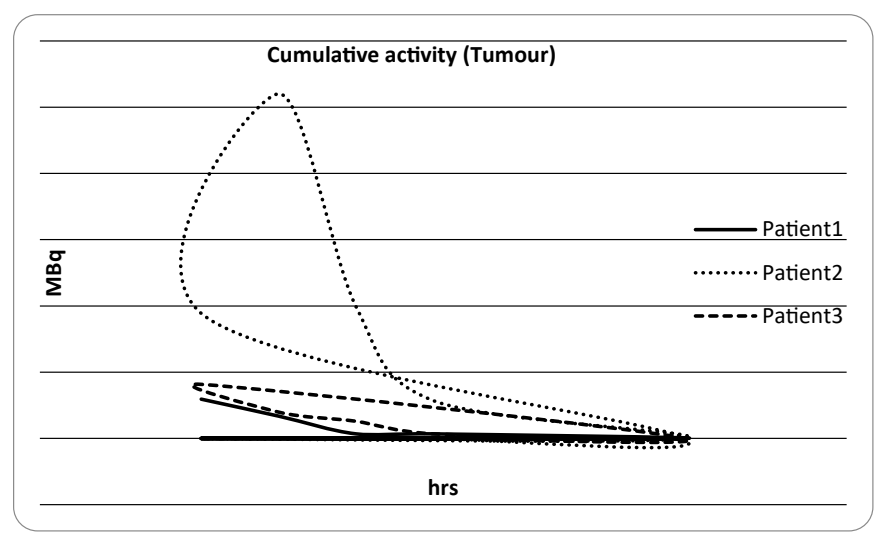

(a)

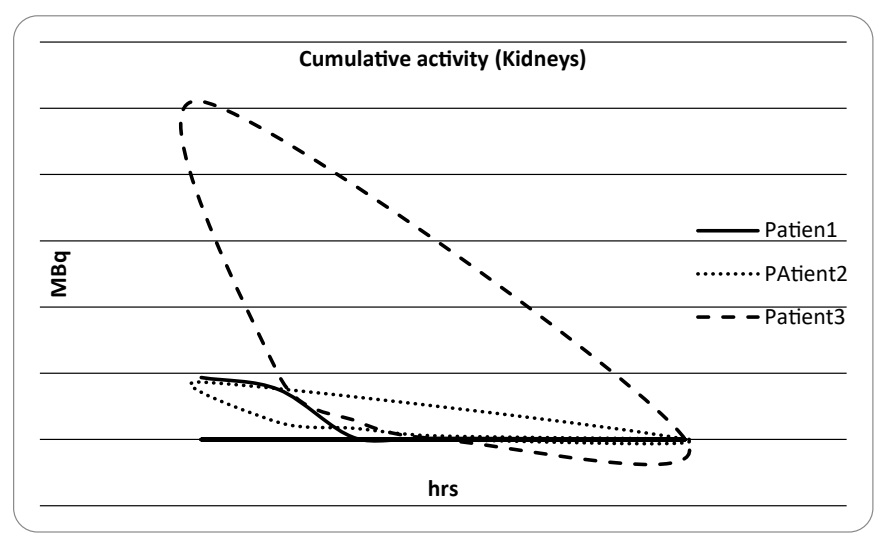

(b)

\section{Discussion}

Questions need to be answered, related to molecular targeted radionuclide therapies, as of how to best extract useful information from nuclear imaging, how to make imaging most quantitative, how to analyse and interpret imaging data. So, the comparison of dosimetric calculations, on a real and a theoretical base is opening doors in declaration of some of the arisen answers, previously mentioned.

Radiopharmaceutical therapy dosimetry differs significantly from the diagnostic applications' dosimetry. The organ absorbed dose is high at radiopharmaceutical therapy and there is a much greater risk for the

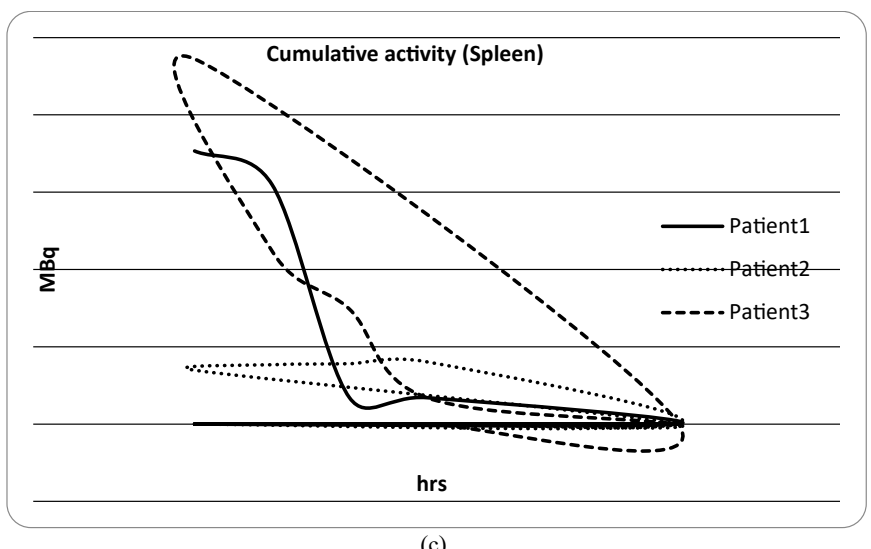

(c)

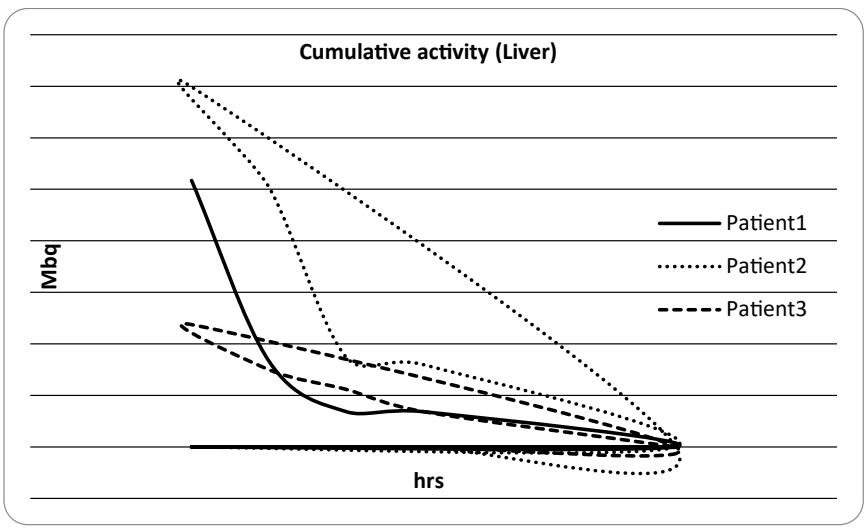

(d)

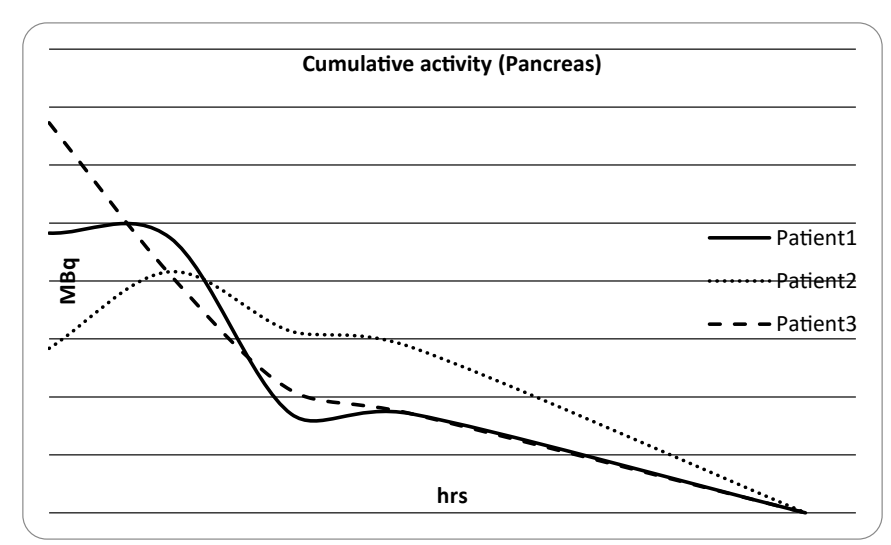

(e)

Figure 3. Cumulative activity curves for tumor, kidney, spleen, liver and pancreas extrapolated to 100 hours (smooth line fit) for each organ, indicatively for three of our patients 


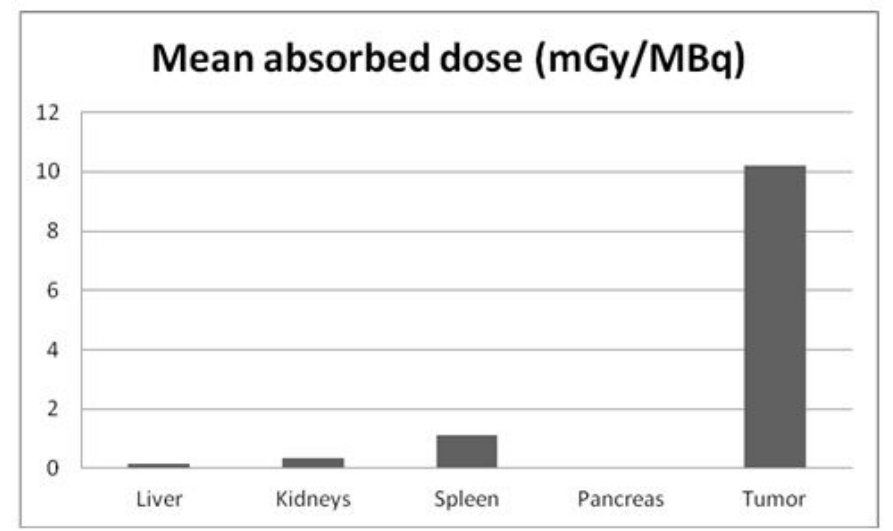

Figure 4. Mean absorbed dose for tumor, kidneys, spleen, liver and pancreas

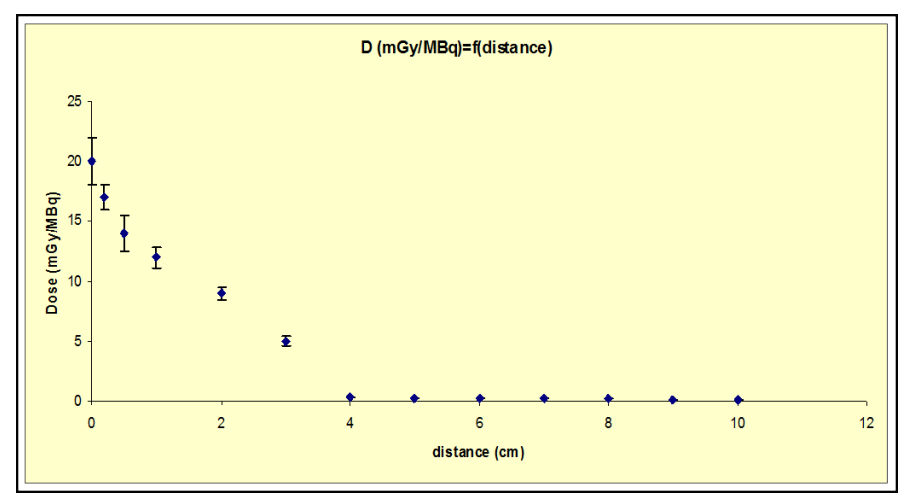

Figure 5. Monte Carlo estimated absorbed dose in the tumor

healthy tissue's healthy tissues. Calculations based on the MIRD schema give a satisfying precision at dose estimation for diagnostic applications, but it is not the same for therapeutic dose estimations. Patient-specific dose calculation is essential for radionuclide therapy because it encompasses individual anatomical details and biophysical processes.

Individualized planar data calculations remain the method of choice in internal dosimetry in nuclear medicine, but with the disadvantage of attenuation and scatter corrections lack and organ overlay. Concerning this study, high deviations of absorbed dose between patients were observed. This proves that patient specific dosimetry must be introduced in radiopharmaceutical therapy department, routinely, as a tool for the determination of the therapeutic activity dose that will have maximum biological effect to the tumour and avoid great risks for the healthy tissue

Furthermore, the wide range of tumor doses could be likely related to biologic and pathologic factors-differences in tumor volume, hypoxia, necrosis, viability, interstitial pressure, heterogeneity in binding affinity, and the receptor density. The latter seems to strongly affect the tumor response during ${ }^{111}$ Indium octreotide therapy.

Optimal results could be obtained with the use of quantitative single-photon emission CT (SPECT) data which are more accurate and allows a patient-specific approach [20-21]. Of course, a theoretical estimation of the accuracy of the results will be achieved with a Monte Carlo simulation.

\section{References}

1. Krenning EP, Kooij PP, Pauwels S, Breeman WA, Postema PT, et al. (1996) Somatostatin receptor: scintigraphy and radionuclide therapy. Digestion 57: 57-61. [Crossref]
2. Krenning EP, Kwekkeboom DJ, Bakker WH, Breeman WA, Kooij PP, et al. (1993) Somatostatin receptor scintigraphy with [111In-DTPA-D-Phe1] - and [123I-Tyr3]octreotide: the Rotterdam experience with more than 1000 patients. Eur J Nucl Med 20: 716-731. [Crossref]

3. Krenning EP, de Jong M, Kooij PP, Breeman WA, Bakker WH, et al. (1999) Radiolabelled somatostatin analogue(s) for peptide receptor scintigraphy and radionuclide therapy. Ann Oncol 10: S23-9. [Crossref]

4. Limouris, G. S. et al. (2005) Evaluation of the therapeutic response to In-111-DTPAoctreotide based targeted therapy in liver metastatic neuroendocrine tumors according to CT/MRI/US findings. Cancer Biother Radiopharm 20: 215-217. [Crossref]

5. Bolch WE, Bouchet LG, Robertson JS, Wessels BW, Siegel JA, et al. (1999) MIRD Pamphlet No. 17: the Dosimetry of nonuniform activity distribution - radionuclide $\mathrm{S}$ values at the voxel level. J NucI Med 40: 11S-36S. [Crossref]

6. Eary JF, Collins C, Stabin M, Vernon C, Petersdorf S, et al. (1993) Samarium-153EDTMP biodistribution and dosimetry estimation. J Nucl Med 34: 1031-1036. [Crossref]

7. Stabin MG, Kooij PP, Bakker WH, Inoue T, Endo K, et al. (1997) Radiation dosimetry for indium-111-pentetreotide. J Nucl Med 38: 1919-1922. [Crossref]

8. Lyra M, Phinou P, Gerlach N, Limouris G, Shukla SK (1998) Absorbed dose calculations in Auger electron therapy of hepatocellural carcinoma by In-111pentetreotide. Radionucl Oncolo Curr Stat Fut Aspcts Med Pub 63-67.

9. ICRP (1998) Radiation Dose to Patients from Radiopharmaceuticals. ICRP Publication 80, International Commission on Radiological Protection. Pergamon Press.

10. ICRP (2008) Radiation dose to patients from radiopharmaceuticals. Addendum 3 to ICRP Publication 53. ICRP Publication 106. Approved by the Commission in October 2007. Ann ICRP 38: 1-197. [Crossref]

11. Kontogeorgakos DK, Dimitriou PA, Limouris GS, Vlahos LJ (2006) Patient specific dosimetry calculation using mathematic models of different anatomic sizes during therapy with ${ }^{111}$ In-DTPAD-Phe1-octreotide infusions after catheterization of the hepatic artery. J Nucl Med 47: 1476-1482. [Crossref]

12. Vamvakas I, Lagopati N, Andreou M, Sotiropoulos M, Gatzis A, et al. (2009) Patient specific computer automated dosimetry calculations during therapy with ${ }^{111}$ In octreotide. Eur J Radiogr 1: 180-183.

13. Lyra M, Phinou P (2000) Internal dosimetry in nuclear medicine: a summary of its development. Applications and current limitations. RSO Mag 5: 17-30.

14. Siegel JA1, Thomas SR, Stubbs JB, Stabin MG, Hays MT, et al. (1999) MIRD pamphlet no. 16, techniques for quantitative radiopharmaceutical biodistribution data acquisition and analysis for use in human radiation dose estimates. $J$ Nucl Med 40 37S-61S. [Crossref]

15. de la Grandmaison GL1, Clairand I, Durigon M (2001) Organ weight in 684 adult autopsies: new tables for a Caucasoid population. Forensic Sci Int 119: 149-154. [Crossref]

16. Inoue Y, Ohtake T, Yoshikawa K, Nishikawa J, Sasaki Y (1998) Estimation of deadtime in imaging human subjects. Eur J Nucl Med 25: 1232-1237. [Crossref]

17. Snyder WS, Ford MR, Warner GG, Watson SB (1975) MIRD pamphlet no. 11, 'S' absorbed dose per unit cumulated activity for selected radionuclides and organs. Medical Intern Dose Comm Soci Nucl Med.

18. Dillman LT,Von der Lage FC (1975) MIRD pamphlet no, 10, radionuclide decay schemes and nuclear parameters for use in radiation dose estimation. Medical Intern Dose Comm Soci Nucl Med.

19. Snyder WS, Ford MR, Warner, GG (1978) MIRD pamphlet no. 5, estimates of specific absorbed fractions for photon sources uniformly distributed in various organs of a heterogeneous phantom. Medical Intern Dose Comm Soci Nucl Med.

20. Flux G, Bardies M, Monsieurs M, Savolainen S, Strands SE, et al. (2006) The impact of PET and SPECT on dosimetry for targeted radionuclide therapy. Z Med Phys 16: 47-59. [Crossref]

21. Kolbert KS, Sgouros G, Scott AM, Bronstein JE, Malane RA, et al. (1997) Implementation and evaluation of patient-specific three-dimensional internal dosimetry. J Nucl Med 38: 301-308. [Crossref]

Copyright: (C2018 Argyrou M. This is an open-access article distributed under the terms of the Creative Commons Attribution License, which permits unrestricted use, distribution, and reproduction in any medium, provided the original author and source are credited. 